\title{
GÊNERO ARCOBACTER E SAÚDE PÚBLICA: revisão sobre sua importância na saúde humana, principais vias de transmissão e patogenicidade
}

\author{
Arcobacter genus and Public Health: review of its \\ importance in human health, main transmission routes \\ and pathogenicity
}

Lauren Machado Moreira ${ }^{10}$; Julia Rosin da Silva ${ }^{1}$; Débora Rodrigues Silveira ${ }^{1 *}$; Cláudio Dias Timm²

*Autor Correspondente: Débora Rodrigues Silveira. Universidade Federal de Pelotas, Campus Universitário, Capão do Leão, s/no, prédio 34, CP 354, Pelotas, RS, Brasil. CEP: 96.001-970. E-mail: debora.rsilveira@hotmail.com

Como citar: MOREIRA, L. M. et al. Gênero Arcobacter e Saúde Pública: revisão sobre sua importância na saúde humana, principais vias de transmissão e patogenicidade. Revista de Educação Continuada em Medicina Veterinária e Zootecnia do CRMV-SP, São Paulo, v. 19, n. 1, 2021, e38112. DOl: https://doi.org/10.36440/ recmvz.v19i1.38112.

Cite as: MOREIRA, L. M. et al. Arcobacter genus and Public Health: review of its importance in human health, main transmission routes and pathogenicity. Journal of Continuing Education in Veterinary Medicine and Animal Science of CRMV-SP, São Paulo, v. 19, n. 1, 2021, e38112. DOl: https://doi.org/10.36440/recmvz. v19i1.38112.

\section{Resumo}

É apresentada uma revisão de literatura sobre o gênero Arcobacter, tratando da sua ocorrência determinando doença em humanos, da sua presença no meio ambiente e em alimentos de origem animal contaminados, bem como dos seus prováveis fatores de patogenicidade. Estes microrganismos estão dispersos no meio ambiente, sendo comumente encontrados em animais de produção e produtos de origem animal. Já foram descritos surtos decorrentes da infecção humana a partir do consumo de carne de frango, suína e leite contaminados. Este agente, além de possuir fatores de adesão e invasão para colonizar o trato gastrintestinal, produz citotoxinas que induzem o organismo do hospedeiro à resposta inflamatória.

Palavras-chave: Doenças Transmitidas por Alimentos. Gastroenterite. Patógeno Negligenciado.

1 Discente de mestrado, Universidade Federal de Pelotas, Departamento de Veterinária Preventiva, Programa de Pósgraduação em Veterinária, Pelotas, RS, Brasil.

2 Docente da Faculdade de Veterinária e orientador do Programa de Pós-graduação em Veterinária, Universidade Federal de Pelotas, Departamento de Veterinária Preventiva, Pelotas, RS, Brasil 


\section{Abstract}

This is a review on the Arcobacter genus, highlighting its occurrence causing disease in humans, and also its presence in the environment and in contaminated animal foods, as well what is known about its probable pathogenicity factors. These microorganisms are dispersed in the environment, being commonly found in farm animals and animal products. Outbreaks of human infection from the consumption of contaminated chicken, pork and milk have been described. This agent, presents adhesion and invasion factors for gastrointestinal tract colonization and produces cytotoxins that induces the inflammation of the host's organism.

Keywords: Foodborne Diseases. Gastroenteritis. Neglected Pathogen.

\section{Introdução}

Arcobacter foi isolado, pela primeira vez, de fetos bovinos e suínos, no final do século XX (ELLIS et al., 1978). No entanto, o gênero Arcobacter foi descrito apenas por Vandamme et al. (1991). Este gênero pertence à família Campylobacteriaceae, a qual compreende dois outros gêneros: Campylobacter e Sulfurospirillum (VANDAMME; DE LEY, 1991).

Arcobacter inclui espécies que são consideradas enteropatógenos emergentes e importantes agentes zoonóticos, uma vez que algumas espécies podem atuar em enteropatias associadas ao consumo de alimentos de origem animal (CHIEFFI; FANELLI; FUSCO, 2020; COLLADO; FIGUERAS, 2011; HO; LIPMAN; GAASTRA, 2006; VAN DEN ABEELE et al., 2014). A. butzleri, A. cryaerophilus, A. cibarus e A. skirrowii são potenciais patógenos humanos e foram isolados de casos de gastroenterite, endocardite, peritonite e bacteremia, bem como de humanos saudáveis (COLLADO; FIGUERAS, 2011; HO; LIPMAN; GAASTRA, 2006; LEVICAN et al., 2015).

Dentre as espécies de Arcobacter relacionadas com doenças transmitidas por alimentos (DTA), estão A. butzleri, A. cryaerophilus e A. skirriwii. A. butzleri, que é a mais frequente, foi isolada de pacientes com quadros diarreicos, bacteremia, peritonite e endocardite, bem como, de suínos, frangos, bovinos, primatas, produtos de origem animal e água contaminada (ANDERSON et al., 1993; SHRESTHA et al., 2019). Além disso, A. butzleri foi recentemente isolada de carnes de frangos e suínos provenientes de açougues (OLIVEIRA et al., 2015), bem como de pescado e ambiente aquático (FANELLI et al., 2019; LAISHRAM et al., 2016).

A. skirrowii foi identificada em amostras oriundas de fetos abortados, prepúcio de touros e cachaços e do útero e ovidutos de fêmeas com problemas reprodutivos (FERNÁNDEZ et al., 1995). A A. cryaerophilus foi isolada de carcaças de frango, e de casos, mastite bovina, bem como de fetos bovinos abortados (FERNÁNDEZ et al., 1995).

Barboza et al. (2017) identificaram A. cryaerophilus em amostras de diarreia humana e Oliveira et al. (2016) isolaram A. cryaerophilus e A. butzleri de jacarés destinados ao abate para consumo humano. Giacometti et al. (2015) isolaram A. cryaerophilus, A. butzleri e A. skirrowii de gado leiteiro, do ambiente de ordenha e A. butzleri também foi identificada em amostras de leite cru.

Infecções por A. butzleri e A. cryaerophilus podem determinar uma variedade de sintomas, desde diarreia até bacteremia (FIGUERAS et al., 2014). Os sinais clínicos apresentados em infecções por A. butzleri e Campylobacter jejuni mostram-se muito semelhantes, porém, o principal sinal clínico associado à infecção por A. butzleri é a diarreia aquosa, que difere das infecções por $C$. jejuni, caracterizadas, principalmente, por diarreia sanguinolenta (VANDENBERG et al., 2004).

Os fatores de patogenicidade de Arcobacter spp. ainda não estão bem definidos, porém alguns autores consideram que os seus mecanismos de patogenicidade envolvem a adesão e invasão das células, estando relacionados principalmente com os genes ciaB, cadF e cj1349, além da produção de citotoxinas que induzem a resposta inflamatória (COLLADO; FIGUERAS, 2011; FERNÁNDEZ et al., 1995; 
FERREIRA; OLEASTRO; DOMINGUES, 2019; HO; LIPMAN; GAASTRA, 2006) e produção de alfa hemolisinas (ATABAY et al., 2002).

Apesar de existir um grande número de casos de gastrenterites provocadas por Campylobacter spp. em humanos, há poucos estudos a respeito de Arcobacter spp. Isto pode ser consequência de não ter sido determinada a causa de infecções em casos em que Campylobacter spp. e outras bactérias similares não foram identificadas. Portanto, o presente trabalho é uma revisão de literatura sobre 0 gênero Arcobacter, destacando a sua ocorrência em humanos, no meio ambiente e em alimentos de origem animal, bem como dos seus prováveis fatores de patogenicidade.

\section{Características do gênero Arcobacter}

O gênero Arcobacter abrange bactérias que inicialmente pertenciam ao gênero Campylobacter devido ao fato de serem microrganismos em forma de espiral, Gram negativos e aerotolerantes, no entanto, posteriormente, constituíram um gênero à parte por possuírem características atípicas de Campylobacter, como capacidade de crescer em temperaturas mais baixas $\left(15^{\circ} \mathrm{C}\right.$ a $\left.30^{\circ} \mathrm{C}\right)$ e sem a necessidade de condições de microaerofilia (PHILLIPS, 2001; SNELLING et al., 2006; VANDAMME et al., 1992). 0 gênero Arcobacter é caracterizado por bactérias que se apresentam como bastonetes curvos, na forma de $\mathrm{S}$ ou helicoidais, não formadoras de esporos e com flagelos polares que the conferem motilidade. São catalases negativas ou fracamente positivas, oxidase positivas, com temperatura de crescimento entre 10 e $37^{\circ} \mathrm{C}$, capazes de se multiplicar em isolamento primário em microaerofilia e, em condições posteriores, em aerobiose, além de apresentarem crescimento variável na presença de 1,5\% a 3,5\% de NaCl (KIEHLBAUCH et al., 1991; KJELDGAARD; JORGENSEN; INGMER, 2009; VANDAMME et al., 1991).

A. butzleri sobrevive em água do mar em estado viável, mas não cultivável, o que permite a sua sobrevivência na ausência de nutrientes e em baixas temperaturas (FERA et al., 2008). Desta maneira, as bactérias do gênero Arcobacter podem sobreviver a $4{ }^{\circ} \mathrm{C}$, temperatura comumente encontrada em abatedouros de frangos e outras espécies. Também podem formar biofilmes (ATANASSOVA et al., 2008; FERREIRA et al., 2013; GIRBAU et al., 2017; KJELDGAARD; JORGENSEN; INGMER, 2009) que favorecem a sobrevivência do agente nos locais contaminados.

Investigações sobre a sobrevivência de Arcobacter spp. em alimentos têm revelado que algumas espécies podem sobreviver em carne suína crua (D'SA; HARRISON, 2005; FERREIRA et al., 2016), em produtos de carne bovina fresca (JASSIM; AL-ABODI, 2020), carne embalada a vácuo (BALAMURUGAN; AHMED; CHAMBERS, 2013) e em leite bovino tanto cru quanto UHT ou pasteurizado (GIACOMETTI et al., 2014), inclusive com grande diversidade genética (MARTA et al., 2020). Também, foi demonstrado que Arcobacter spp. sobrevive durante a produção ou armazenamento de muçarela de búfala (SERRAINO et al., 2013), em ricota (GIACOMETTI et al., 2015) e na água em diferentes temperaturas normalmente utilizadas na indústria de alimentos (VAN DRIESSCHE; HOUF, 2007).

Cervenka et al. (2008) e Silha et al. (2014) observaram que Arcobacter spp. sobrevive em superfícies de aço inoxidável, plástico e vidro, Rasmussen et al. (2013) relataram a tolerância do Arcobacter spp. a alguns biocidas, como hipoclorito de sódio, comumente empregado na desinfecção em indústria. O A. butzleri pode sobreviver aos procedimentos comuns de higienização (HAUSDORF et al., 2013; RASMUSSEN et al., 2013; SCARANO et al., 2014). As informações relacionadas à sobrevivência e à permanência de Arcobacter spp. durante o processamento dos alimentos, bem como em toda a cadeia alimentar até os produtos serem oferecidos ao consumidor final, demonstram a sua participação como patógeno para seres humanos.

Arcobacter tornou-se um grande grupo de bactérias, e a intensificação do seu estudo determinou a descrição de uma diversidade de novas espécies. Atualmente estão descritas e reconhecidas 26 espécies provenientes de diversos ambientes e hospedeiros como por exemplo: Arcobacter porcinus, 
descrita a partir do Arcobacter thereius (FIGUERAS et al., 2016), Arcobacter lekithochrous, isolado obtido de um incubatório de moluscos (DIÉGUEZ et al., 2017) e Arcobacter canalis, isolado de moluscos e água de um canal contaminado com esgoto urbano (CATALUÑA; SALAS-MASSÓ; FIGUERAS, 2018).

\section{Infecção por Arcobacter spp.}

Os veículos mais frequentes de transmissão de Arcobacter para humanos são a água potável contaminada e alimentos crus ou malcozidos que são ingeridos ou manipulados (DUFFY; FEGAN, 2012; VANDAMME et al., 1992). Outras possíveis formas de contágio são a transmissão direta entre seres humanos durante um surto (VANDAMME et al., 1992), contaminação de feridas e fístulas (HSUEH et al., 1997) e transmissão transplacentária ao feto (ON; STACEY; SMYTH, 1995).

O Arcobacter spp. já foi isolado de lençóis freáticos durante o período em que ocorreu um surto em seres humanos (FONG et al., 2007).

Um surto de doença causada pelo Arcobacter spp. associado ao consumo de frango assado foi relatado em um casamento nos EUA, onde 51 de 109 convidados apresentaram sintomas como diarreia, dores abdominais, fadiga, náusea, calafrios, dores no corpo e dor de cabeça. Cinco pacientes forneceram amostras de fezes e em quatro delas foi identificado A. butzleri, indicando que esse microrganismo pode causar doença veiculada por alimentos (LAPPI et al., 2013).

Petersen et al. (2007), Houf et al. (2008), Fera et al. (2009) e Giacometti et al. (2015) destacam que o contato com animais de estimação também pode ser uma forma de transmissão do Arcobacter spp. para humanos pois cães e gatos podem estar infectados por este agente.

O principal sintoma decorrente da infecção de seres humanos por Arcobacter spp. é a diarreia aquosa persistente, porém em muitos casos, a enterite clínica resultante da infecção é autolimitante (HO; LIPMAN; GAASTRA, 2006).

As espécies de Arcobacter associadas a doença em seres humanos são A. butzleri, A. cryaerophilus e A. skirrowii (OLIVEIRA et al., 1997). O A. butzleri é a principal espécie do gênero relacionada às implicações em saúde pública (COLLADO; FIGUERAS, 2011).

Estudos epidemiológicos, relatos de casos e relatos de surtos têm constatado que A. butzleri e A. cryaerophilus estão relacionadas a casos de enterite em seres humanos, com isolamento do agente em amostras de fezes diarreicas (BARBOZA et al., 2017; FERNÁNDEZ; KRAUSE; VILLANUEVA, 2004; FERREIRA et al., 2016; FIGUERAS et al., 2014; KAYMAN et al., 2012; LAPPI et al., 2013; LEHNER; TASARA; STEPHAN, 2005; TEE et al., 1988; VANDAMME et al., 1992). A infecção por este agente foi associada à colite e, até mesmo, à septicemia (LAU et al., 2002), porém o A. cryaerophilus já foi isolado de humanos assintomáticos (HOUF; STEPHAN, 2007). A. butzleri foi isolado, na Turquia, em 1,25\% de amostras de fezes diarreicas (KAYMAN et al., 2012) e na Nova Zelândia, A. butzleri e A. cryaerophilus foram isoladas de 0,9\% de amostras de fezes (MANDISODZA; BURROWS; NULSEN, 2012). Em Portugal, o Arcobacter também foi identificado em seres humanos com diarreia, onde 1,3\% das amostras foram positivas para A. butzleri e 0,3\% para A. cryaerophilus (FERREIRA et al., 2014). Collado et al. (2013), em Valdivia, no sul do Chile, observaram uma prevalência de $1,4 \%$ para $A$. butzleri em amostras oriundas de diarreia, sem detecção em amostras de pacientes assintomáticos.

A. skirrowii foi o agente causador de bacteremia e colite em relatos de casos individuais na Catalunha, nordeste da Espanha (COLLADO; GUARRO; FIGUERAS, 2009), e em casos de diarreia crônica e aguda em humanos, na Bélgica (WYBO; BREYNAERT; LAUWERS, 2004). Acredita-se que os humanos possam se infectar pelas via fecal-oral, ingestão de água ou de alimentos de origem animal contaminados e por contato direto com animais infectados (COLLADO; FIGUERAS, 2011; LEHNER; TASARA; STEPHAN, 2005; WESLEY; MILLER, 2010).

Embora Arcobacter spp. não represente um agente de destacada importância em Saúde Pública, o crescente aumento de casos sugere que a sua importância deva ser repensada no contexto das 
zoonoses e das doenças veiculadas por alimentos e água. É possível que a sua importância como agente etiológico de zoonose e doença transmitida por alimentos possa estar sendo subestimada, principalmente pela escassez de métodos de detecção e identificação (FIGUERAS; COLLADO; GUARRO, 2008; SNELLING et al. 2006; VANDENBERG et al. 2004).

\section{Arcobacter spp. em produtos de origem animal e água}

Em carnes, a maior prevalência do Arcobacter spp. tem sido verificada em frangos, seguida de suínos e bovinos. Em países como Austrália, Holanda e Itália, a sua presença foi confirmada em carcaças de frangos em mais de 70\% das amostras analisadas (ÇELIK; OTLU, 2020; HO; LIPMAN; GAASTRA, 2008; PENTAMALLI et al., 2009; RIVAS; FEGAN; VANDERLINDE, 2004). No Brasil, Oliveira et al. (2015) isolaram a bactéria em 18,3\% (55/300) de amostras de carne de frango provenientes de açougues, das quais 63,6\% (35/55) foram identificadas como A. butzleri e 36,3\% (20/55) como A. cryaerophilus. A alta densidade populacional no criatório e as falhas nas boas práticas de abate, mantendo, por exemplo, o contato entre carcaças são fatores que contribuem para a contaminação da carne de frango. Tais condições favorecem uma rápida disseminação de qualquer patógeno que possa ter acesso ao criatório (MEAD, 2004).

Em suínos, o primeiro isolamento de Arcobacter spp. se deu em carne moída, nos EUA, em 1994 (COLLINS; WESLEY; MURANO, 1996). No Brasil, o Arcobacter spp. foi isolado de amostras de estômago de suínos abatidos em um frigorífico no Rio Grande do Sul, das quais 79\% (98/148) foram identificadas como A. cryaerophilus e $21 \%$ (26/148) como A. butzleri (VOGT et al., 2008). Destaque-se que $A$. butzleri e A. cryaerophilus são resistentes à temperatura de escaldagem em abatedouros ( $62 \circ \mathrm{C}$ a $72^{\circ} \mathrm{C}$ ) durante três minutos (HO; LIPMAN; GAASTRA, 2008), o que pode favorecer a sua persistência e disseminação nos procedimentos de abate e processamento das carcaças.

No Chile, Fernández et al. (2015) observaram altas frequências de Arcobacter spp. em carne de frango pronta para consumo humano (92\%) e de A. butzleri em suínos, 40,7\% (55/135) e em bovinos 26,7\% (20/75). Já para $A$. cryaerophylus, foram encontradas frequências de 9,6 (13/135) e 6,7\% (5/75) para suínos e bovinos, respectivamente. Na Austrália, Duffy e Fegan (2012) isolaram Arcobacter spp. em 83,3\% de 130 carcaças bovinas amostradas em quatro diferentes matadouros.

A presença de Arcobacter spp. no trato intestinal de animais saudáveis pode representar risco de contaminação de carcaças durante o abate (DE SMET; DE ZUTTER; HOUF, 2011; SHAH et al., 2011). Além da contaminação da cadeia alimentar, a contaminação do meio ambiente também pode ser uma consequência da infecção por Arcobacter spp. devido a sua presença nas fezes, o que resultaria na contaminação do solo e das fontes de água (CHINIVASAGAM et al., 2007; WILKES et al., 2014). A contaminação cruzada entre carcaças ou por meio de equipamentos e meio ambiente durante o processamento de abate também foi atribuída como causa da contaminação dos alimentos (FERREIRA et al., 2013; HO; LIPMAN; GAASTRA, 2008; SHANGE; GOUWS; HOFFMAN, 2019; VAN DRIESSCHE; HOUF, 2007).

Pianta et al. (2007) relataram o isolamento de Arcobacter spp. de amostras de leite bovino (6/32), onde cinco foram identificadas como A. cryaerophilus e uma como A. butzleri. 0 Arcobacter spp. foi identificado em amostras de leite cru bovino, que são frequentemente contaminadas por $A$. butzleri (8/10) (GIACOMETTI et al., 2015).

O Arcobacter foi isolado de jacarés, do pantanal do Brasil, abatidos para consumo humano, com registro de $37,5 \%$ (15/40) das amostras positivas para A. butzleri e 50\% (20/40) para A. cryaerophylus (OLIVEIRA et al., 2016).

O Arcobacter spp. foi detectado em ovos, com 0,8\% (4/475) de amostras positivas (LEE et al., 2016). Carne de cordeiro, de coelho e de avestruz também podem apresentar contaminação por Arcobacter spp. (COLLADO; GUARRO; FIGUERAS, 2009; RAHIMI, 2014; RIVAS; FEGAN; VANDERLINDE, 2004).

Frutos do mar e mariscos têm apresentando alta prevalência de positividade para diversas espécies de Arcobacter (COLLADO; GUARRO; FIGUERAS, 2009; DIÉGUEZ et al., 2017; LEVICAN et al., 2014, 
2015; MOTTOLA et al., 2016; NIEVA-ECHEVARRIA et al., 2013; CATALUÑA; SALAS-MASSÓ; FIGUERAS, 2018; SILHA; SILHOVA-HRUSKOVA; VYTRASOVA, 2015). Mottola et al. (2016) detectaram a presença de Arcobacter spp. em mariscos, com 23\% (16/70) de isolamentos, dos quais 75\% (12/16) foram positivos para A. butzleri e 25\% (4/16) para A. cryaerophilus.

Arcobacter spp. foram isolados de alimentos já preparados, em restaurantes de Bangkok, com uma prevalência superior a outros patógenos como Salmonella e Campylobacter (TEAGUE, 2010).

o Arcobacter spp. pode contaminar o meio aquático como mares, lagos e rios, bem como a água de abastecimento em abatedouros, casas e criatórios, estações de tratamento e efluentes de esgoto (COLLADO et al., 2008; DIERGAARDT et al., 2004). Giacometti et al. (2015) propõem que a água pode ser a via de transmissão do Arcobacter spp. para os animais, resultando em sua ampla disseminação no ambiente agrícola, não sendo portanto, a contaminação fecal a única via de contaminação do leite.

\section{Fatores de patogenicidade de Arcobacter spp.}

Dentre as espécies de Arcobacter, as que são consideradas patogênicas para o ser humano são A. butzleri, A. cryaerophilus e A. skirrowii (NEWELL, 1997). Apesar de inúmeros estudos estarem sendo realizados, a patogenicidade e os fatores de virulência de Arcobacter spp. ainda não estão bem definidos. Alguns autores acreditam que os seus mecanismos de patogenicidade envolvam adesão e invasão das células hospedeiras e produção de toxinas (FERNÁNDEZ et al., 1995; HO; LIPMAN; GAASTRA, 2006). Devido à semelhança dos sinais clínicos das infecções por Campylobacter spp. e Arcobacter spp., acredita-se que alguns fatores de virulência de Campylobacter jejuni e Campylobacter coli também estariam presentes nas espécies patogênicas de Arcobacter (DOUIDAH et al., 2012).

Collado et al. (2010) estudaram a invasão, a adesão e a capacidade citotóxica de quatro espécies de Arcobacter (A. butzleri, A. cryaerophilus, A. skirrowii e A. cibarius), usando linhagens de células HEp-2, HeLa, INT407, CHO e Caco-2, constatando que diferentes espécies apresentaram consideráveis variações na adesão, invasão e citotoxicidade, dependendo dos isolados e das células estudadas.

No sequenciamento genômico de A. butzleri ATCC 49616, foi confirmada a presença de genes determinantes de virulência, como cadF, cj1349 e ciaB, que codificam as proteínas de ligação da fibronectina, possibilitando a adesão da bactéria às células epiteliais do intestino do hospedeiro (FLANAGAN et al., 2009; KONKEL et al., 1999). 0 gene cadF também induz a introdução da bactéria na célula eucariótica pela ativação de GTPases e o gene ciaB contribui para a invasão das células hospedeiras. Também foi identificado o gene $\operatorname{irgA}$, que está relacionado à aquisição de ferro pela célula bacteriana, por meio da codificação de uma proteína da membrana, bem como os genes hecA, hecB e tlyA, responsáveis pela codificação da hemoaglutinina filamentosa associada à adesão, à ativação da proteína da hemolisina e à sua codificação, respectivamente. Por fim, também foram identificados o gene pldA associado à lise de eritrócitos, e o fator de virulência mviN associado à capacidade de evasão do sistema imune inato (DASTI et al., 2010; MILLER et al., 2007).

Levican et al. (2013) identificaram 60 isolados de Arcobacter spp., dos quais 51 apresentaram o gene ciaB, seguido do gene cj1349 (23/60) e cadF (15/60). Cinquenta e duas estirpes de $A$. butzleri foram analisadas com o emprego de PCR (KARADAS et al., 2013). Os genes ciaB, mviN, pldA, tlyA, cj1349 e cadF foram detectados em todos os isolados, enquanto $\operatorname{irgA}(15 \%)$, hecB (44\%), hecA (13\%) foram detectados apenas em alguns. Quatro de seis isolados foram testados quanto à invasão em células HT-29 e três foram capazes de invadi-las, e os mesmos seis isolados testados apresentaram capacidade de adesão e invasão em células Caco-2. Ainda foram sequenciados os genes ciaB, cadF e cj1349 de seis isolados, e não foram observadas alterações significativas nos domínios funcionais de aminoácidos.

No Brasil, a presença de A. butzleri foi verificada na fase final do abate de frangos e suínos, totalizando 155 cortes de carne suína e 105 cortes de carne de frango, e o isolamento de Arcobacter 
spp. foi obtido em 12 cortes suínos e cinco cortes em aves. Os genes de pldA, cadF, ciaB e cj1349 estavam presentes em todos os isolados de origem suína e aviária (OLIVEIRA et al., 2015).

Com relação à patogenicidade e aos fatores de virulência de Arcobacter spp., ainda não existe uma conclusão definitiva. Diversos genes encontrados são homólogos aos presentes em espécies de Campylobacter, Salmonella entérica sorovar Thiphimurim, Sulfurimonas denitrificans e Wolinella succinogenes, porém ainda é incerto se são funcionais ou desempenham um papel similar (MILLER et al., 2007; OHNISHI et al., 1990).

\section{Considerações finais}

Muitas espécies pertencentes ao gênero Arcobacter têm sido isoladas de diversas origens, desde o ambiente até os animais de produção, bem como de inúmeros alimentos de origem animal, e, consequentemente, de humanos com alterações no trato gastrointestinal. A sintomatologia apresentada por humanos infectados também ocorre quando da infecção por bactérias da família Campylobacteriaceae. É provável que muitos casos de doença veiculada por alimentos tenham tido o Arcobacter spp. como agente, porém ele não foi considerado como uma das suspeitas e não foi investigado.

Considerando-se as dificuldades de eliminação do Arcobacter ssp. em plantas frigoríficas, devem ser pesquisados e empregados sanitizantes em concentrações suficientes para controlar a contaminação das superfícies, evitando assim a disseminação do microrganismo durante o processamento dos produtos. Ainda sobre pesquisas futuras sugere-se o estudo minucioso dos seus fatores de virulência como adesão, invasão das células hospedeiras e produção de toxinas para ser definitivamente determinado o funcionamento de tais mecanismos e assim serem devolvidas formas de combate às espécies patogênicas. Concomitantemente, deve ser ampliada a divulgação da ocorrência Arcobacter spp. entre profissionais da saúde, centros de diagnóstico, órgãos regulamentadores de alimentos, os próprios controles de qualidades de produtos de origem animal das indústrias e consumidores para evitar a negligência aos agravos que ele determina.

\section{Referências}

ANDERSON, K. F. et al. Arcobacter (Campylobacter) butzleri-associated diarrheal illness in a nonhuman primate population. Infection and Immunity, [s.I.], v. 61, n. 5, p. 2220-2223, 1993. DOI: https://doi. org/10.1128/iai.61.5.2220-2223.1993.

ATABAY, H. I. et al. Discrimination of Arcobacter butzleri isolates by polimerase chain reactionmediated DNA fingerprinting. Letters in Applied Microbiology, [s.I.], v. 35, n. 2, p. 141-145, 2002. DOI: https://doi.org/10.1046/j.1472-765x.2002.01152.x.

ATANASSOVA, V. et al. Incidence of Arcobacter spp. in poultry: quantitative and qualitative analysis and PCR differentiation. Journal of food protection, v. 71, n. 12, p. 2533-2536, 2008.

BALAMURUGAN, S.; AHMED, R.; CHAMBERS, J. R. Survival of Arcobacter butzleri on vacuum packaged chill stored beef. Food Research International, [s.I.], v. 52, n. 2, p. 503-507, 2013. DOl: https://doi. org/10.1016/J.FOODRES.2013.01.048.

BARBOZA, K. et al. First isolation report of Arcobacter cryaerophilus from a human diarrhea sample in Costa Rica. Revista do Instituto de Medicina Tropical de São Paulo, [s.l.], v. 59, p. 1-5, 2017. DOI: https://doi.org/10.1590/S1678-9946201759072.

CATALUÑA, A. P.; SALAS-MASSÓ, N.; FIGUERAS, M. J. Arcobacter canalis sp. nov., isolated from a water canal contaminated with urban sewage. International Journal of Systematic and Evolutionary

Microbiology, [s.I.], v. 68, n. 4, p. 1258-1264, 2018. DOl: https://doi.org/10.1099/ijsem.0.002662. 
ÇELIK, E.; OTLU, S. Isolation of Arcobacter spp. and identification of isolates by multiplex PCR from various domestic poultry and wild avian species. Annals of Microbiology, [s.I.], v. 70, n. 1, p. 1-7, 2020. DOl: https://doi.org/10.1186/s13213-020-01603-7.

CERVANKA, L. et al. Persistence of Arcobacter butzleri CCUG 30484 on plastic, stainless steel and glass surfaces. Brazilian Journal of Microbiology, [s.I.], v. 39, n. 3, p. 517-520, 2008. DOI: https://doi. org/10.1590/S1517-83822008000300021.

CHIEFFI, D.; FANELLI, F.; FUSCO, V. Arcobacter butzleri: Up-to-date taxonomy, ecology, and pathogenicity of an emerging pathogen. Comprehensive Reviews in Food Science and Food Safety, [s.l.], v. 19, n. 4, p. 2071-2109, 2020. DOl: https://doi.org/10.1111/1541-4337.12577.

CHINIVASAGAM, H. N. et al. Detection of Arcobacter spp. in piggery effluent and effluent-irrigated soils in southeast Queensland. Journal of Applied Microbiology, [s.l.], v. 103, n. 2, p. 418-426, 2007. DOI: https://doi.org/10.1111/j.1365-2672.2007.03275.x.

COLLADO, L. et al. Assessment of the prevalence and diversity of emergent campylobacteria in human stool samples using a combination of traditional and molecular methods. Diagnostic Microbiology and Infectious Disease, [s.I.], v. 75, n. 4, p. 434-436, 2013. DOI: https://doi.org/10.1016/j. diagmicrobio.2012.12.006.

COLLADO, L. et al. Presence of Arcobacter spp. in environmental waters correlates with high levels of fecal pollution. Environmental Microbiology, [s.I.], v. 10, n. 6, p. 1635-1640, 2008. DOI: https://doi. org/10.1111/j.1462-2920.2007.01555.x.

COLLADO, L.; FIGUERAS, M. J. Taxonomy, epidemiology and clinical relevance of the genus Arcobacter. Clinical Microbiology Reviews, [s.I.], v. 24, n. 1, p. 174-192, 2011. DOI: https://doi.org/10.1128/ CMR.00034-10.

COLLADO, L.; GUARRO, J.; FIGUERAS, M. J. Prevalence of Arcobacter in meat and shellfish. Journal of Food Protection, [s.I.], v. 72, n. 5, p. 1102-1106, 2009. DOl: https://doi.org/10.4315/0362028x-72.5.1102.

COLLADO, L. et al. Taxonomy and epidemiology of the genus Arcobacter. 2010. Tese (Doutorado) Rovira i Virgili University, Reus, Spain, 2010.

COLLINS, C. I.; WESLEY, I. V.; MURANO, E. A Detection of Arcobacter spp. in ground pork by modified plating methods. Journal of Food Protection, [s.I.], v. 59, n. 5, p. 448-452, 1996. DOl: https://doi. org/10.4315/0362-028X-59.5.448.

DASTI, J. I. et al. Campylobacter jejuni: a brief overview on pathogenicity-associated factors and disease-mediating mechanisms. International Journal of Medical Microbiology, [s.I.], v. 300, n. 4, p. 205-211, 2010. DOI: https://doi.org/10.1016/j.jjmm.2009.07.002.

D'SA, E. M.; HARRISON, M. A. Effect of $\mathrm{pH}, \mathrm{NaCl}$ content, and temperature on growth and survival of Arcobacter spp. Journal of Food Protection, [s.I.], v. 68, n. 1, p. 18-25, 2005. DOl: https://doi. org/10.4315/0362-028x-68.1.18.

DE SMET, S.; DE ZUTTER, L.; HOUF, K. Small ruminants as carriers of the emerging foodborne pathogen Arcobacter on small and medium farms. Small Ruminant Research, [s.l.], v. 97, n. 1-3, p. 124-129, 2011. DOI: https://doi.org/10.1016/j.smallrumres.2011.02.004.

DIÉGUEZ, A. L. et al. Arcobacter lekithochrous sp. nov., isolated from a molluscan hatchery. International Journal of Systematic and Evolutionary Microbiology, [s.l.], v. 67, n. 5, p. 13271332, 2017. DOI: https://doi.org/10.1099/ijsem.0.001809.

DIERGAARDT, S. M. et al. The occurrence of campylobacters in water sources in South Africa. Water Research, [s.l.], v. 38, n. 10, p. 2589-2595, 2004. DOI: https://doi.org/10.1016/j. watres.2004.03.004. 
DOUIDAH, L. et al. Occurrence of putative virulence genes in Arcobacter species isolated from humans and animals. Journal of Clinical Microbiology, [s.I.], v. 50, n. 3, p. 735-741, 2012. DOl: https://doi. org/10.1128/JCM.05872-11.

DUFFY, L. L.; FEGAN, N. Prevalence and concentration of Arcobacter spp. on Australian beef carcasses. Journal of Food Protection, [s.I.], v. 75, n. 8, p. 1479-1482, 2012. DOI: https://doi. org/10.4315/0362-028X.JFP-12-093.

ELLIS, W. A. et al. Isolation of spirillum-like organisms from pig fetuses. The Veterinary Record, [s.I.], v. 102, n. 5, p. 106, 1978. DOl: https://doi.org/10.1136/vr.102.5.106-a.

FANELLI, F. et al. Genomic characterization of Arcobacter butzleri isolated from shellfish: novel insight into antibiotic resistance and virulence determinants. Frontiers in Microbiology, [s.I.], v. 10, p. 670, 2019. DOl: https://doi.org/10.3389/fmicb.2019.00670.

FERA, M. T. et al. Induction and resuscitation of viable noncultivable Arcobacter butzleri cells. Applied and Environmental Microbiology, [s.I.], v. 74, n. 10, p. 3266-3268, 2008. DOI: https://doi. org/10.1128/AEM.00059-08.

FERA, M. T. et al. Pet cats as carriers of Arcobacter spp. in Southern Italy. Journal of Applied Microbiology, [s.I.], v. 106, n. 5, p. 1661-1666, 2009. DOl: https://doi.org/10.1111/j.1365-2672.2008.04133.x.

FERNÁNDEZ, H. et al. Arcobacter butzleri and A. cryaerophilus in human, animals and food sources, in southern Chile. Brazilian Journal of Microbiology, [s.I.], v. 46, n. 1, p. 145-147, 2015. DOI: https:// doi.org/10.1590/S1517-838246120140095.

FERNÁNDEZ, H. et al. Toxigenic and invasive capacities: possible pathogenic mechanisms in Arcobacter cryaerophilus. Memórias do Instituto Oswaldo Cruz, [s.I.], v. 90, n. 5, p. 633-634, 1995. DOI: https:// doi.org/10.1590/S0074-02761995000500018.

FERNÁNDEZ, H.; KRAUSE, S.; VILLANUEVA, M. P. Arcobacter butzleri an emerging enteropathogen: communication of two cases with chronic diarrhea. Brazilian Journal of Microbiology, [s.l.], v. 35, n. 3, p. 216-218, 2004. DOI: https://doi.org/10.1590/S1517-83822004000200008.

FERREIRA, S. et al. Genetic diversity, antibiotic resistance and biofilm-forming ability of Arcobacter butzleri isolated from poultry and environment from a Portuguese slaughterhouse. International Journal of Food Microbiology, [s.l.], v. 162, n. 1, p. 82-88, 2013. DOI: https://doi.org/10.1016/j. ijfoodmicro.2013.01.003.

FERREIRA, S. et al. Insights in the pathogenesis and resistance of Arcobacter: A review. Critical Reviews in Microbiology, [s.l.], v. 42, n. 3, p. 364-383, 2016. DOI: https://doi.org/10.3109/1040841X.2014.954523.

FERREIRA, S. et al. Molecular diagnosis of Arcobacter and Campylobacter in diarrhoeal samples among Portuguese patients. Diagnostic Microbiology and Infectious Disease, [s.I.], v. 78, n. 3, p. 220-225, 2014. DOI: https://doi.org/10.1016/j.diagmicrobio.2013.11.021.

FERREIRA, S.; OLEASTRO, M.; DOMINGUES, F. Current insights on Arcobacter butzleri in food chain. Current Opinion in Food Science, [s.I.], v. 26, p. 9-17, 2019. DOI: https://doi.org/10.1016/j.cofs.2019.02.013.

FIGUERAS, M. J.; COLLADO, L.; GUARRO, J. A new 165 rDNA-RFLP method for the discrimination of the accepted species of Arcobacter. Diagnostic Microbiology and Infectious Disease, [s.I.], v. 62, n. 1, p. 11-15, 2008. DOl: https://doi.org/10.1016/j.diagmicrobio.2007.09.019.

FIGUERAS, M. J. et al. Arcobacter ellisii sp. nov., isolated from mussels. Systematic and Applied Microbiology, [s.l.], v. 34, n. 6, p. 414- 418, 2011. DOl: https://doi.org/10.1016/j.syapm.2011.04.004.

FIGUERAS, M. J. et al. 'Arcobacter porcinus' sp. nov., a novel Arcobacter species uncovered by Arcobacter thereius. New Microbes New Infections, [s.I.], v. 15, p. 104-106, 2016. DOI: https://doi. org/10.1016/j.nmni.2016.11.014. 
FIGUERAS, M. J. et al. A severe case of persistent diarrhea associated with Arcobacter cryaerophilus but attributed to Campylobacter sp. and a review of the clinical incidence of Arcobacter spp. New Microbes and New Infections, [s.I.], v. 2, n. 2, p. 31-37, 2014. DOl: https://doi.org/10.1002/2052-2975.35.

FLANAGAN, R. C. et al. Examination of Campylobacter jejuni putative adhesins leads to the identification of a new protein, designated flpA, required for chicken colonization. Infection and Immunity, [s.I.], v. 77, n. 6, p. 2399-2407, 2009. DOl: https://doi.org/10.1128/IAl.01266-08.

FONG, T. T. et al. Massive microbiological groundwater contamination associated with a waterborne outbreak in Lake Erie, South Bass Island, Ohio. Environmental Health Perspectives, [s.I.], v. 115, n. 6, p. 856-864, 2007. DOI: https://doi.org/10.1289/ehp.9430.

GIACOMETTI, F. et al. Arcobacter butzleri, Arcobacter cryaerophilus, and Arcobacter skirrowii circulation in a dairy farm and sources of milk contamination. Applied and Environmental Microbiology, [s.l.], v. 81, n. 15, p. 5055-5063, 2015. DOl: https://doi.org/10.1128/AEM.01035-15.

GIACOMETTI, F. et al. Behavior of Arcobacter butzleri and Arcobacter cryaerophilus in ultrahightemperature, pasteurized, and raw cow's milk under different temperature conditions. Foodborne Pathogens and Disease, [s.I.], v. 11, n. 1, p. 15-20, 2014. DOl: https://doi.org/10.1089/fpd.2013.1597.

GIACOMETTI, F.; LOSIO, M. N.; SERREINO, A. Short communication: Arcobacter butzleri and Arcobacter cryaerophilus survival and growth in artisanal and industrial ricotta cheese. Journal of Dairy Science, [s.I.], v. 98, n. 10, p. 6776-6781, 2015. DOl: https://doi.org/10.3168/jds.2015-9560.

GIRBAU, C. et al. Study of biofilm formation ability of foodborne Arcobacter butzleri under different conditions. Journal of Food Protection, [s.I.], v. 80, n. 5, p. 758-762, 2017. DOI: https://doi. org/10.4315/0362-028X.JFP-16-505.

HAUSDORF, L. et al. Occurrence and genetic diversity of Arcobacter spp. in a spinach-processing plant and evaluation of two Arcobacter-specific quantitative PCR assays. Systematic and Applied Microbiology, [s.I.], v. 36, n. 4, p. 235-243, 2013. DOl: https://doi.org/10.1016/j. syapm.2013.02.003.

HO, H. T. K.; LIPMAN, L. J. A.; GAASTRA, W. Arcobacter, what is known and unknown about a potential food borne zoonotic agent! Veterinary Microbiology, [s.I.], v. 115, n. 1-3, p. 1-13, 2006. DOI: https:// doi.org/10.1016/j.vetmic.2006.03.004.

HO, H. T. K.; LIPMAN, L. J. A.; GAASTRA, W. The introduction of Arcobacter spp. in poultry slaughterhouses. International Journal of Food Microbiology, [s.I.], v. 125, n. 3, p. 223-229, 2008. DOI: https://doi. org/10.1016/j.jifoodmicro.2008.02.012.

HOUF, K. et al. Dogs as carriers of the emerging pathogen Arcobacter. Veterinary Microbiology, [s.l.], v. 130, n. 1-2, p. 208-213, 2008. DOI: https://doi.org/10.1016/j.vetmic.2008.01.006.

HOUF, K.; STEPHAN, R. Isolation and characterization of the emerging foodborne pathogen Arcobacter from human stool. Journal of Microbiological Methods, [s.l.], v. 68, n. 2, p. 408-413, 2007. DOI: https://doi.org/10.1016/j.mimet.2006.09.020.

HSUEH, P. R. et al. Bacteremia caused by Arcobacter cryaerophilus 1B. Journal Clinical Microbiology, [s.l.], v. 35, n. 2, p. 489-491, 1997. DOl: https://doi.org/10.1128/jcm.35.2.489-491.1997.

JASSIM, S. A.; AL-ABODI, H. R. Resistance rate and novel virulence factor determinants of Arcobacter spp., from cattle fresh meat products from Iraq. Microbial Pathogenesis, [s.l.], v. 152, e104649, 2020. DOI: https://doi.org/10.1016/j.micpath.2020.104649.

KARADAS, G. et al. Presence of virulence genes, adhesion and invasion of Arcobacter butzleri. Journal of Applied Microbiology, [s.l.], v. 115, n. 2, p. 583-590, 2013. DOl: https://doi.org/10.1111/

jam.12245. 
KAYMAN, T. et al. Emerging pathogen Arcobacter spp. in acute gastroenteritis: molecular identification, antibiotic susceptibilities and genotyping of the isolated arcobacters. Journal of Medical Microbiology, [s.l.], v. 61, n. 10, p. 1439-1444, 2012. DOI: https://doi.org/10.1099/jmm.0.044594-0.

KAYMAN, T. et al. Human acute gastroenteritis associated with Arcobacter butzleri. Clinical Microbiology Newsletter, [s.I.], v. 34, n. 24, p. 197-199, 2012a. D0l: https://doi.org/10.1016/j. clinmicnews.2012.11.004.

KIEHLBAUCH, J. A. et al. Restriction fragment length polymorphisms in the ribosomal genes for species identification and subtyping of aerotolerant Campylobacter species. Journal Clinical Microbiology, [s.I.], v. 29, n. 8, p. 1670-1676, 1991. DOl: https://doi.org/10.1128/jcm.29.8.1670-1676.1991.

KJELDGAARD, J.; JORGENSEN, K.; INGMER, H. Growth and survival at chiller temperatures of Arcobacter butzleri. International Journal of Food Microbiology, [s.I.], v. 131, n. 2-3, p. 256-259, 2009. DOI: https://doi.org/10.1016/j.ijfoodmicro.2009.02.017.

KONKEL, M. E. et al. Identification of the enteropathogens Campylobacter jejuni and Campylobacter coli based on the cadF virulence gene and its product. Journal of Clinical Microbiology, [s.I.], v. 37, n. 3, p. 510-517, 1999. DOl: https://doi.org/10.1128/JCM.37.3.510-517.1999.

LAISHRAM, M. et al. Isolation and characterization of Arcobacter spp. from fresh seafood and the aquatic environment. International Journal of Food Microbiology, [s.l.], v. 232, p. 87-89, 2016. DOI: https://doi.org/10.1016/j.ijfoodmicro.2016.05.018.

LAPPI, V. et al. An outbreak of foodborne illness among attendees of a wedding reception in Wisconsin likely caused by Arcobacter butzleri. Foodborne Pathogens Disease, [s.I.], v. 10, n. 3, p. 250-255, 2013. DOI: https://doi.org/10.1089/fpd.2012.1307.

LAU, S. K. P. et al. Identification by $16 \mathrm{~s}$ ribosomal DNA gene seqencing of Arcobacter butzleri bacteraemia in a patient with acute gangrenous appendicitis. Molecular Pathology, [s.I.], v. 55, n. 3, p. 182-185, 2002. DOI: https://doi.org/10.1136/mp.55.3.182.

LEE, M. et al. Detection of foodborne pathogens and mycotoxins in eggs and chicken feeds from farms to retail markets. Korean Journal for Food Science of Animal Resources, [s.I.], v. 36, n. 4, p. $463-$ 468, 2016. DOI: https://doi.org/10.5851/kosfa.2016.36.4.463.

LEHNER, A.; TASARA, T.; STEPHAN, R. Relevant aspects of Arcobacter spp. as potential foodborne pathogen. International Journal of Food Microbiology, [s.I.], v. 102, n. 2, p. 127-135, 2005. DOI: https://doi.org/10.1016/j.ijfoodmicro.2005.03.003.

LEVICAN, A. et al. Adherence to and invasion of human intestinal cells by Arcobacter species and their virulence genotypes. Applied Environmental of Microbiology, [s.I.], v. 79, n. 16, p. 4951-4957, 2013. DOI: https://doi.org/10.1128/AEM.01073-13.

LEVICAN, A. et al. Arcobacter ebronensis sp. nov. and Arcobacter aquimarinus sp. nov., two new species isolated from marine environment. Systematic and Applied Microbiology, [s.I.], v. 38, n. 1, p. 30-35, 2015. DOI: https://doi.org/10.1016/j.syapm.2014.10.011

LEVICAN, A. et al. Higher water temperature and incubation under aerobic and microaerobic conditions increase the recovery and diversity of Arcobacter spp. from shellfish. Applied and Environmental Microbiology, [s.I.], v. 80, n. 1, p. 385-391, 2014. DOl: https://doi.org/10.1128/AEM.03014-13. MANDISODZA, O.; BURROWS, E.; NULSEN, M. Arcobacter species in diarrhoeal faeces from humans in New Zealand. New Zealand Medical Journal, [s.l.], v. 125, n. 1353, p. 40-46, 2012.

MARTA, C. et al. Large genetic diversity of Arcobacter butzleri isolated from raw milk in Southern Italy. Food Microbiology, [s.l.], v. 89, e103403, 2020. DOl: https://doi.org/10.1016/j.fm.2019.103403. 
MEAD, G. C. Microbiological quality of poultry meat: a review. Revista Brasileira Ciência Avícola, [s.l.], v. 6, n. 3, p. 135-142, 2004. DOl: https://doi.org/10.1590/S1516-635X2004000300001.

MILLER, W. G. et al. The complete genome sequence and analysis of the epsilon proteobacterium Arcobacter butzleri. PLoS One, [s.l.], v. 2, n. 12, e1358, 2007. DOl: https://doi.org/10.1371/journal. pone.0001358.

MOTTOLA, A. et al. Occurrence of potentially pathogenic arcobacters in shellfish. Food Microbiology, [s.I.], v. 57, p. 23-27, 2016. DOI: https://doi.org/10.1016/j.fm.2015.12.010.

NEWELL, D. G. Campylobacters, Helicobacters and Related organisms: disease associations in pigs. The Pig Journal, [s.I.], v. 39, p. 102, 1997.

NIEVA-ECHEVARRIA, B. et al. Prevalence and genetic diversity of Arcobacter in food products in the north of Spain. Journal of Food Protection, [s.I.], v. 76, n. 8, p. 1447-1450, 2013. DOI: https://doi. org/10.4315/0362-028X.JFP-13-014.

OHNISHI, K. et al. Gene fliA encodes an alternative sigma factor specific for flagellar operons in Salmonella typhimurium. Molecular Genetics and Genomics, [s.I.], v. 221, n. 2, p. 139-147, 1990. DOI: https://doi.org/10.1007/BF00261713.

OLIVEIRA, M. G. X. et al. Detecção de estirpes virulentas de Arcobacter butzleri em carnes de frangos e suínos provenientes de açougues no município de São Paulo (projeto em andamento). Revista de Educação Continuada em Medicina Veterinária e Zootecnia do CRMV-SP, [s.I.], v. 13, n. 1, p. 89-90, 2015.

OLIVEIRA, M. G. X. et al. Monitoramento de estirpes virulentas de Arcobacter spp. em jacarés (Caiman yacare) destinados ao abate e consumo. Revista de Educação Continuada em Medicina Veterinária e Zootecnia do CRMV-SP, [s.I.], v. 14, n. 1, p. 81-82, 2016.

OLIVEIRA, S. J. et al. Classification of Arcobacter species isolated from aborted pig fetuses and sows with reproductive problems in Brazil. Veterinary Microbiology, [s.I.], v. 57, n. 4, p. 347-354, 1997. DOI: https://doi.org/10.1016/s0378-1135(97)00106-5.

ON, S. L.; STACEY, A.; SMYTH, J. Isolation of Arcobacter butzleri from a neonate with bacteraemia. Journal of Infection, [s.l.], v. 31, n. 3, p. 225-227, 1995. DOI: https://doi.org/10.1016/s01634453(95)80031-X.

PENTIMALLI, D. et al. Specific PCR detection of Arcobacter butzleri, Arcobacter cryaerophilus, Arcobacter skirrowii, and Arcobacter cibarius in chicken meat. Journal of Food Protection, [s.I.], v. 72, n.7, p. 1491-1495, 2009. DOI: https://doi.org/10.4315/0362-028x-72.7.1491.

PETERSEN, R. F. et al. A PCR-DGGE method for detection and identification of Campylobacter, Helicobacter, Arcobacter and related Epsilobacteria and its application to saliva samples from humans and domestic pets. Journal of Applied Microbiology, [s.I.], v. 103, n. 6, p. 2601-2615, 2007. DOI: https://doi.org/10.1111/j.1365-2672.2007.03515.x.

PHILLIPS, C. A. Arcobacter spp. in food: isolation, identification and control. Trends in Food Science and Technology, [s.l.], v. 12, n. 8, p. 263-275, 2001. DOl: https://doi.org/10.1016/S0924-2244(01)00090-5. PIANTA, C. et al. Isolation of Arcobacter spp. from the milk of dairy cows in Brazil. Ciência Rural, [s.l.], v. 37, n. 1, p. 171-174, 2007. DOl: https://doi.org/10.1590/50103-84782007000100027.

RAHIMI, E. Prevalence and antimicrobial resistance of Arcobacter species isolated from poultry meat in Iran. British Poultry Science, [s.I.], v. 55, n. 2, p. 174-180, 2014. DOI: https://doi.org/10.1080/0007 1668.2013 .878783 .

RASMUSSEN, L. H. et al. Multilocus sequence typing and biocide tolerance of Arcobacter butzleri from Danish broiler carcasses. BMC Research Notes, [s.I.], v. 6, p. 1-7, 2013. 
RIVAS, L.; FEGAN, N.; VANDERLINDE, P. Isolation and characterisation of Arcobacter butzleri from meat. International Journal of Food Microbiology, [s.I.], v. 91, n. 1, p. 31-41, 2004. D0I: https://doi. org/10.1016/S0168-1605(03)00328-3.

SAMIE, A. et al. Prevalence of Campylobacter species, Helicobacter pylori and Arcobacter species in stool samples from the Venda region, Limpopo, South Africa: studies using molecular diagnostic methods. Journal of Infection, [s...], v. 54, n. 6, p. 558-566, 2007. DOl: https://doi.org/10.1016/j.jinf.2006.10.047.

SCARANO, C. et al. Arcobacter butzleri in sheep ricotta cheese at retail and related sources of contamination in an industrial dairy plant. Applied and Environmental Microbiology, [s.l.], v. 80, n. 22, p. 7036-7041, 2014. DOI: https://doi.org/10.1128/AEM.02491-14.

SERRAINO, A. et al. Survival of Arcobacter butzleri during production and storage of artisan water buffalo mozzarella cheese. Foodborne Pathogens and Disease, [s.l.], v. 10, n. 9, p. 820-824, 2013. DOI: https://doi.org/10.1089/fpd.2013.1485.

SHAH, A. H. et al. Arcobacter: an emerging threat to animals and animal origin food products? Trends in Food Science and Technology, [s.I.], v. 22, n. 5, p. 225-236, 2011. DOI: https://doi.org/10.1016/j. tifs.2011.01.010.

SHANGE, N.; GOUWS, P.; HOFFMAN, L. C. Campylobacter and Arcobacter species in food-producing animals: prevalence at primary production and during slaughter. World Journal of Microbiology and Biotechnology, [s.I.], v. 35, n. 9, p. 146, 2019. DOl: https://doi.org/10.1007/s11274-019-2722-x.

SHRESTHA, R. G. et al. Prevalence of Arcobacter and other pathogenic bacteria in river water in Nepal. Water, [s.l.], v. 11, n. 7, p. 1416, 2019. DOI: https://doi.org/10.3390/w11071416.

SILHA, D. et al. Survival of selected bacteria from the genus Arcobacter on various metallic surfaces. Journal of Food and Nutrition Research, [s.I.], v. 53, n. 3, p. 217-223, 2014.

SILHA, D.; SILHOVA-HRUSKOVA, L.; VYTRASOVA, J. Modified isolation method of Arcobacter spp. from different environmental and food samples. Folia Microbiologica, [s.l.], v. 60, n. 6, p. 515-521, 2015. DOI: https://doi.org/10.1007/s12223-015-0395-x.

SNELLING, W. J. et al. Under the microscope: Arcobacter. Letters in Applied Microbiology, [s.I.], v. 42, n. 1, p. 7-14, 2006. DOI: https://doi.org/10.1111/j.1472-765X.2005.01841.X.

TEAGUE, N. S. et al. Enteric pathogen sampling of tourist restaurants in Bangkok, Thailand. Journal Travel Medicine, [s.l.], v. 17, p. 118-123, 2010.

TEE, W. et al. Campylobacter cryaerophila isolated from a human. Journal of Clinical Microbiology, [s.I.], v. 26, n. 12, p. 2469-2473, 1988.

VAN DEN ABEELE, A. et al. Prevalence of Arcobacter species among humans, Belgium, 20082013. Emerging Infectious Diseases, [s.I.], v. 20, n. 10, p. 1731-1734, 2014. DOI: https://doi. org/10.3201/eid2010.140433.

VANDAMME, P.; DE LEY, J. Proposal for a New Family, Campylobacteraceae. International Journal of Systematic and Evolutionary Microbiology, [s.I.], v. 41, n. 3, p. 451-455, 1991. DOI: https://doi. org/10.1099/00207713-41-3-451.

VANDAMME, P. et al. Outbreak of recurrent abdominal cramps associated with Arcobacter butzleri in an Italian school. Journal of Clinical Microbiology, [s.l.], v. 30, n. 9, p. 2335-2337, 1992. DOI: https:// doi.org/10.1128/jcm.30.9.2335-2337.1992.

VANDAMME, P. et al. Revision of Campylobacter, Helicobacter, and Wolinella taxonomy: emendation of generic descriptions and proposal of Arcobacter gen. nov. International Journal of Systematic and Evolutionary Microbiology, [s.I.], v. 41, n. 1, p. 88-103, 1991. DOI: https://doi. org/10.1099/00207713-41-1-88. 
VANDENBERG, O. et al. Arcobacter species in humans. Emerging Infectious Diseases, [s.I.], v. 10, n. 10, p. 1863-1867, 2004. DOl: https://doi.org/10.3201/eid1010.040241.

VAN DRIESSCHE, E.; HOUF, K. Characterization of the Arcobacter contamination on Belgian pork carcasses and raw retail pork. International Journal of Food Microbiology, [s.I.], v. 118, n. 1, p. 20-26, 2007. DOI: https://doi.org/10.1016/j.ijfoodmicro.2007.05.006.

VAN DRIESSCHE, E.; HOUF, K. Survival capacity in water of Arcobacter species under different temperature conditions. Journal of Applied Microbiology, [s.I.], v. 105, n. 2, p. 443-451, 2008. DOI: https://doi.org/10.1111/j.1365-2672.2008.03762.x.

VOGT, F. I. et al. Cultivo de Arcobacter spp. a partir de diferentes graus de lesões de úlcera gástrica em suínos. Revista de Iniciação Científica da ULBRA, [s.I.], n. 7, p. 21-34, 2008.

WESLEY, I. V.; MILLER, G. W. Arcobacter: an opportunistic human food-borne pathogen? In: SCHELD, W. M.; GRAYSON, M. L.; HUGHES, J. M. (ed.). Emerging infections 9. Washington, D. C.: ASM Press, 2010. p. 185-211.

WILKES, G. et al. Long-term monitoring of waterborne pathogens and microbial source tracking markers in paired agricultural watersheds under controlled and conventional tile drainage management. Applied and Environmental Microbiology, [s.I.], v. 80, n. 12, p. 3708-3720, 2014. DOI: https://doi.org/10.1128/AEM.00254-14.

WYBO, I.; BREYNAERT, J.; LAUWERS, S. Isolation of Arcobacter skirrowii from a patient with chronic diarrhea. Journal of Clinical Microbiology, [s.I.], v. 42, n. 4, p. 1851-1852, 2004. DOI: https://doi. org/10.1128/JCM.42.4.1851-1852.2004. 